\title{
The Cauchy problem for intuitionistic fuzzy differential equations
}

\section{Bouchra Ben Amma, Said Melliani and Lalla Saadia Chadli}

\author{
Laboratory of Applied Mathematics and Scientific Competing (LMACS) \\ Sultan Moulay Slimane University \\ PO Box 523, 23000 Beni Mellal, Morocco \\ e-mails: bouchrabenamma@gmail .com, s.melliani@yahoo.fr, \\ sa.chadli@yahoo.fr
}

Received: 20 October 2017 Revised: 4 December $2017 \quad$ Accepted: 6 December 2017

\begin{abstract}
In this paper we discuss the existence and uniqueness theorem of a solution of the cauchy problem of intuitionistic fuzzy differential equation.
\end{abstract}

Keywords: Intuitionistic fuzzy differential equations, Approximate solutions, Existence, Uniqueness.

2010 Mathematics Subject Classification: $03 E 72$.

\section{Introduction}

One of the generalizations of fuzzy sets theory [15] can be considered the proposed intuitionistic fuzzy sets(IFS). Later on Atanassov generalized the concept of fuzzy set and introduced the idea of intuitionistic fuzzy set [1-3]. They are very necessary and powerful tool in modeling imprecision, valuable applications of IFSs have been flourished in many different field $[4,6,8,9]$.

For intuitionistic fuzzy concepts, recently the authors [5,11-13] established, the theory of metric space of intuitionistic fuzzy sets, intuitionistic fuzzy differential equations, intuitionistic fuzzy fractional equation and the Cauchy problem for complex intuitionistic fuzzy differential equations. They proved the existence and uniqueness of the intuitionistic fuzzy solution for these intuitionistic fuzzy differential equations using different concepts. 
This paper is to investigate the existence and uniqueness theorem of intuitionistic fuzzy solutions for the follwing intuitionistic fuzzy differential equations:

$$
\left(\langle u, v\rangle^{\prime}(t)=f\left(t,(\langle u, v\rangle(t)), \quad\langle u, v\rangle\left(t_{0}\right)=\left\langle u_{0}, v_{0}\right\rangle\right.\right.
$$

when $\left\langle u_{0}, v_{0}\right\rangle$ is an intuitionistic fuzzy quantity and $f$ satisfies the generalized Lipschitz condition.

The paper is organized as follows. In Section 2, we collect the fundamental notions and facts which will be used in the rest of the article and we list several comparison propositions on classical ordinary differential equations in [7]. In Section 3 we show the relation between a solution and its approximate solution to the Cauchy problem of the intuitionistic fuzzy differential equation, and furthermore, in Section 4, we prove the existence and uniqueness theorem for a solution to the Cauchy problem of the intuitionistic fuzzy differential equation.

\section{Preliminaries}

Throughout this paper, $\left(\mathbb{R}^{n}, B\left(\mathbb{R}^{n}\right), \mu\right)$ denotes a complete finite measure space.

Let us $P_{k}\left(\mathbb{R}^{n}\right)$ the set of all non empty compact convex subsets of $\mathbb{R}^{n}$.

we denote by

$$
\mathbb{F}_{n}=\operatorname{IF}\left(\mathbb{R}^{n}\right)=\left\{\langle u, v\rangle: \mathbb{R}^{n} \rightarrow[0,1]^{2}, \mid \forall x \in \mathbb{R}^{n} 0 \leq u(x)+v(x) \leq 1\right\}
$$

An element $\langle u, v\rangle$ of $\mathbb{F}_{n}$ is said an intuitionistic fuzzy number if it satisfies the following conditions

(i) $\langle u, v\rangle$ is normal i.e there exists $x_{0}, x_{1} \in \mathbb{R}^{n}$ such that $u\left(x_{0}\right)=1$ and $v\left(x_{1}\right)=1$.

(ii) $u$ is fuzzy convex and $v$ is fuzzy concave.

(iii) $u$ is upper semi-continuous and $v$ is lower semi-continuous

(iv) $\operatorname{supp}\langle u, v\rangle=\operatorname{cl}\left\{x \in \mathbb{R}^{n}: \mid v(x)<1\right\}$ is bounded.

so we denote the collection of all intuitionistic fuzzy numbers by $\mathbb{F}_{n}$

On the space $\mathbb{F}_{n}$ we will consider the following metric,

$$
\begin{aligned}
& d_{\infty}^{n}(\langle u, v\rangle,\langle z, w\rangle)=\frac{1}{4} \sup _{0<\alpha \leq 1}\left\|[\langle u, v\rangle]_{r}^{+}(\alpha)-[\langle z, w\rangle]_{r}^{+}(\alpha)\right\| \\
& +\frac{1}{4} \sup _{0<\alpha \leq 1}\left\|[\langle u, v\rangle]_{l}^{+}(\alpha)-[\langle z, w\rangle]_{l}^{+}(\alpha)\right\|+\frac{1}{4} \sup _{0<\alpha \leq 1}\left\|[\langle u, v\rangle]_{r}^{-}(\alpha)-[\langle z, w\rangle]_{r}^{-}(\alpha)\right\| \\
& +\frac{1}{4} \sup _{0<\alpha \leq 1}\left\|[\langle u, v\rangle]_{l}^{-}(\alpha)-[\langle z, w\rangle]_{l}^{-}(\alpha)\right\|
\end{aligned}
$$

where $\|$.$\| denotes the usual Euclidean norm in \mathbb{R}^{n}$.

Theorem 2.1 ([12]). $d_{\infty}^{n}$ define a metric on $I F_{n}$.

Theorem 2.2 ([12]). The metric space $\left(I F_{n}, d_{\infty}^{n}\right)$ is complete. 
The norm $\|\quad\|$ of an intuitionistic fuzzy number $\langle u, v\rangle \in I F_{n}$ is defined by

$$
\|\langle u, v\rangle\|=d_{\infty}^{n}\left(0_{(1,0)},\langle u, v\rangle\right)=\left\|[\langle u, v\rangle]_{0}\right\|=\frac{1}{2} \sup _{a \in[\langle u, v\rangle]_{0}}|a|+\frac{1}{2} \inf _{b \in[\langle u, v\rangle]]^{0}}|b|
$$

Definition 2.1. An intuitionistic fuzzy set $\langle u, v\rangle$ is called convex intuitionistic fuzzy set if and only if $u$ is convex fuzzy set and $v$ is concave fuzzy set.

The question that arises, is what $I F_{n}$ with addition and multiplication by a scalar is a vector Theorem 2.3 ([13]). There exists a normed space $X$ and a function $j: I F_{n} \longrightarrow X$ with properties:

1. $j$ is an isometry i.e. $\left\|j(\langle u, v\rangle)-j\left(\left\langle u^{\prime}, v^{\prime}\right\rangle\right)\right\|=d_{\infty}^{n}\left(\langle u, v\rangle,\left\langle u^{\prime}, v^{\prime}\right\rangle\right)$

2. $j\left(\langle u, v\rangle \oplus\left\langle u^{\prime}, v^{\prime}\right\rangle\right)=j(\langle u, v\rangle)+j\left(\left\langle u^{\prime}, v^{\prime}\right\rangle\right)$

3. $j(\lambda\langle u, v\rangle)=\lambda j(\langle u, v\rangle) \quad \lambda \geq 0$

Remark 2.1. if $\langle u, v\rangle(t): T \longrightarrow I F_{n}$ is differentiable at $t_{0} \in T$, then

$$
(j\langle u, v\rangle)(t)=j(\langle u, v\rangle(t)) \quad T \longrightarrow X
$$

is Frechet differentiable at $t_{0}$ and $(j\langle u, v\rangle)^{\prime}\left(t_{0}\right)=j\left(\langle u, v\rangle^{\prime}\left(t_{0}\right)\right)$, where $j$ is the embedding in Theorem 2.3.

In the following we list several comparison propositions on classical ordinary differential equations following [7]

Proposition 2.1. Let $G \subset \mathbb{R}^{2}$ be an open set and $g \in C[G, \mathbb{R}],\left(t_{0}, x_{0}\right) \in G$. Suppose $r(t)$ is the maximum solution to the initial value problem

$$
x^{\prime}=g(t, x), \quad x\left(t_{0}\right)=x_{0}
$$

and its largest interval of existence of right solution is $\left[t_{0}, t_{0}+a\right)$. If $\left[t_{0}, t_{1}\right] \subset\left[t_{0}, t_{0}+a\right)$, then there exists an $\varepsilon_{0}>0$ such that the maximum solution $r(t, \varepsilon)$ to the initial value problem

$$
x^{\prime}=g(t, x)+\varepsilon, \quad x\left(t_{0}\right)=x_{0}+\varepsilon
$$

exists on $\left[t_{0}, t_{1}\right]$ whenever $0<\varepsilon<\varepsilon_{0}$, and $r(t, \varepsilon)$ uniformly converges to $r(t)$ on $\left[t_{0}, t_{1}\right]$ as $\varepsilon \longrightarrow 0^{+}$

Proposition 2.2. Let $G \subset \mathbb{R}^{2}$ be an open set, $g \in C[G, \mathbb{R}],\left(t, x_{0}\right) \in G$. Suppose that the maximum solution to the initial value problem (2.1) is $r(t)$ and its largest interval of existence of right solution is $\left[t_{0}, t_{0}+a\right)$. If $m(t) \in C\left[\left[t_{0}, t_{0}+a\right), \mathbb{R}\right]$, satisfies $(t, m(t)) \in G$ for all $t \in\left[t_{0}, t_{0}+a\right), m\left(t_{0}\right) \leq x_{0}$, and

$$
D m(t) \leq g(t, m(t)), \quad \forall t \in\left[t_{0}, t_{0}+a\right) \backslash \Gamma
$$

where D is one of the four Dini derivatives (see [7]), G at most is a countable set on $t$. Then we must have

$$
m(t) \leq r(t), \quad \forall t \in\left[t_{0}, t_{0}+a\right)
$$




\section{The relation between a solution and its approximate solution to intuitionistic fuzzy differential equations}

Assume that $f: T \times W \longrightarrow I F_{n}$ is continuous (it is denoted by $f \in C\left[T \times W, I F_{n}\right]$ ). Consider the initial value problem

$$
\langle u, v\rangle^{\prime}(t)=f\left(t,(\langle u, v\rangle(t)), \quad\langle u, v\rangle\left(t_{0}\right)=\langle u, v\rangle_{0}\right.
$$

where $W \subset I F_{n},\langle u, v\rangle\left(t_{0}\right) \in W$.

In the following we give the relation between a solution and its approximate solutions. We denote $R_{0}=\left[t_{0}, t_{0}+p\right] \times B\left(\langle u, v\rangle_{0}, q\right)$ where $p>0, q>0,\langle u, v\rangle_{0} \in I F_{n}, B\left(\langle u, v\rangle_{0}, q\right)=$ $\left\{\langle u, v\rangle \in I F_{n} \backslash d_{\infty}^{n}\left(\langle u, v\rangle,\langle u, v\rangle_{0}\right) \leq q\right\}$

Theorem 3.1. Let $f \in C\left[R_{0}, I F_{n}\right], r \in(0, p),\langle u, v\rangle_{n} \in C^{1}\left[\left[t_{0}, t_{0}+r\right], B\left(\langle u, v\rangle_{0}, q\right)\right]$ such that

$$
\begin{array}{r}
j\langle u, v\rangle_{n}^{\prime}(t)=j f\left(t,\langle u, v\rangle_{n}(t)\right)+B_{n}(t),\langle u, v\rangle\left(t_{0}\right)=\langle u, v\rangle_{0}, \\
\left\|B_{n}(t)\right\| \leq \varepsilon_{n} \forall t \in\left[t_{0}, t_{0}+r\right](n=0,1,2, \ldots),
\end{array}
$$

where $\varepsilon_{n}>0, \varepsilon_{n} \longrightarrow 0, B_{n}(t) \in C\left[\left[t_{0}, t_{0}+r\right], X\right]$ and $j$ is the isometric embedding from $\left(I F_{n}, d_{\infty}^{n}\right)$ onto its range in the Banach space $X$. For each $t \in\left[t_{0}, t_{0}+r\right]$ there exist an $\delta(t)>0$ such that H-differences $\langle u, v\rangle_{n}(t+h) \ominus\langle u, v\rangle_{n}(t)$ and $\langle u, v\rangle_{n}(t) \ominus\langle u, v\rangle_{n}(t-h)$ exist for all $0 \leq h<\delta(t)$ and $n=1,2, \ldots$

if we have

$$
d_{\infty}^{n}\left(\langle u, v\rangle_{n}(t),\langle u, v\rangle(t)\right) \longrightarrow 0 \quad \text { u.c } \forall t \in\left[t_{0}, t_{0}+r\right](n \longrightarrow \infty)
$$

(u.c. denotes the uniform convergence), then $\langle u, v\rangle \in C^{1}\left[\left[t_{0}, t_{0}+r\right], B\left(\langle u, v\rangle_{0}, q\right)\right]$

$$
\langle u, v\rangle^{\prime}(t)=f(t,\langle u, v\rangle(t)), \quad\langle u, v\rangle\left(t_{0}\right)=\left\langle u_{0}, v_{0}\right\rangle, \quad t \in\left[t_{0}, t_{0}+r\right] .
$$

Proof 1. From (3.3) we know that $\langle u, v\rangle \in C\left[\left[t_{0}, t_{0}+r\right], B\left(x_{0}, q\right)\right]$. For fixed $t_{1} \in\left[t_{0}, t_{0}+r\right]$ and any $t \in\left[t_{0}, t_{0}+r\right], t>t_{1}$, denote

$$
F(t, n)=\frac{j\langle u, v\rangle_{n}(t)-j\langle u, v\rangle_{n}\left(t_{1}\right)}{t-t_{1}}-j f\left(t_{1},\langle u, v\rangle_{n}\left(t_{1}\right)\right)-B_{n}\left(t_{1}\right)
$$

It is well known that

$$
\lim _{n \longrightarrow \infty} F(t, n)=\frac{j\langle u, v\rangle(t)-j\langle u, v\rangle\left(t_{1}\right)}{t-t_{1}}-j f\left(t_{1},\langle u, v\rangle\left(t_{1}\right)\right)
$$

From $f \in C^{1}\left[R_{0}, I F_{n}\right]$ is known that for any $\varepsilon>0$, there exists $\delta_{1}>0$ such that

$$
d_{\infty}^{n}\left(f(t,\langle z, w\rangle(t)), f\left(t_{1},\langle u, v\rangle\left(t_{1}\right)\right)\right)<\frac{\varepsilon}{4}
$$

whenever $t_{1}<t<t_{1}+\delta_{1}$ and $d_{\infty}^{n}\left(\langle z, w\rangle(t),\langle u, v\rangle\left(t_{1}\right)\right)<\delta_{1}$ with $\left.\langle z, w\rangle \in B\left(\langle u, v\rangle_{0}, q\right)\right]$. Take natural number $N>0$ such hat

$$
\varepsilon_{n}<\frac{\varepsilon}{4}, \quad d_{\infty}^{n}\left(\langle u, v\rangle_{n}(t),\langle u, v\rangle(t)\right)<\frac{\delta_{1}}{2} \quad \text { for any } n>N, t \in\left[t_{0}, t_{0}+r\right] .
$$


Take $\delta>0$ such that $\delta<\delta_{1}$ and

$$
d_{\infty}^{n}\left(\langle u, v\rangle(t),\langle u, v\rangle\left(t_{1}\right)\right)<\frac{\delta_{1}}{2} \quad \text { whenever } t_{1}<t<t_{1}+\delta
$$

By the definition of $F(t, n)$ and (3.2), we have

$$
j\langle u, v\rangle_{n}(t)-j\langle u, v\rangle_{n}\left(t_{1}\right)-\left(t-t_{1}\right) j\langle u, v\rangle_{n}^{\prime}\left(t_{1}\right)=\left(t-t_{1}\right) F(t, n)
$$

We choose $\varphi \in X^{*}$ such that $\|\varphi\|=1$ and

$$
\begin{aligned}
\varphi\left(j\langle u, v\rangle_{n}(t)-j\langle u, v\rangle_{n}\left(t_{1}\right)-\left(t-t_{1}\right) j\langle u, v\rangle_{n}^{\prime}\left(t_{1}\right)\right) \\
=\left\|j\langle u, v\rangle_{n}(t)-j\langle u, v\rangle_{n}\left(t_{1}\right)-\left(t-t_{1}\right) j\langle u, v\rangle_{n}^{\prime}\left(t_{1}\right)\right\|
\end{aligned}
$$

Let $\psi(t)=\varphi\left(j\langle u, v\rangle_{n}(t)\right)-\left(t-t_{1}\right) \varphi\left(j\langle u, v\rangle_{n}^{\prime}\left(t_{1}\right)\right)$, consequently,

$$
\psi^{\prime}(t)=\varphi\left(j\langle u, v\rangle_{n}(t)\right)-\varphi\left(j\langle u, v\rangle_{n}^{\prime}\left(t_{1}\right)\right)
$$

hence

$$
\begin{aligned}
& \left\|j\langle u, v\rangle_{n}(t)-j\langle u, v\rangle_{n}\left(t_{1}\right)-\left(t-t_{1}\right) j\langle u, v\rangle_{n}^{\prime}\left(t_{1}\right)\right\|=\psi(t)-\psi\left(t_{1}\right)=\psi^{\prime}(\bar{t})\left(t-t_{1}\right) \\
= & \varphi\left(j\langle u, v\rangle_{n}^{\prime}(\bar{t})-j\langle u, v\rangle_{n}^{\prime}\left(t_{1}\right)\right)\left(t-t_{1}\right) \leq\|\varphi\| \cdot\left\|j\langle u, v\rangle_{n}^{\prime}(\bar{t})-j\langle u, v\rangle_{n}^{\prime}\left(t_{1}\right)\right\| \cdot\left(t-t_{1}\right) \\
= & \left\|j\langle u, v\rangle_{n}^{\prime}(\bar{t})-j\langle u, v\rangle_{n}^{\prime}\left(t_{1}\right)\right\| \cdot\left(t-t_{1}\right)
\end{aligned}
$$

where $t_{1} \leq \bar{t} \leq t$. In view of (3.9), we have

$$
\|F(t, n)\| \leq\left\|j\langle u, v\rangle_{n}^{\prime}(\bar{t})-j\langle u, v\rangle_{n}^{\prime}\left(t_{1}\right)\right\|, \quad t_{1} \leq \bar{t} \leq t
$$

From (3.7) and (3.8) we know that

$$
\begin{aligned}
& d_{\infty}^{n}\left(\langle u, v\rangle(\bar{t}),\langle u, v\rangle\left(t_{1}\right)\right)<\frac{\delta_{1}}{2} \\
d_{\infty}^{n}\left(\langle u, v\rangle_{n}(\bar{t}),\langle u, v\rangle\left(t_{1}\right)\right) & \leq d_{\infty}^{n}\left(\langle u, v\rangle_{n}(\bar{t}),\langle u, v\rangle(\bar{t})\right)+d_{\infty}^{n}\left(\langle u, v\rangle(\bar{t}),\langle u, v\rangle\left(t_{1}\right)\right) \\
& <\frac{\delta_{1}}{2}+\frac{\delta_{1}}{2}=\delta_{1}
\end{aligned}
$$

Hence by (3.6) and (3.10) we have

$$
\begin{aligned}
\|F(t, n)\| & \leq\left\|j\langle u, v\rangle_{n}^{\prime}(\bar{t})-j\langle u, v\rangle_{n}^{\prime}\left(t_{1}\right)\right\| \\
& =\left\|j f\left(\bar{t},\langle u, v\rangle_{n}(\bar{t})\right)+B_{n}(\bar{t})-j f\left(t_{1},\langle u, v\rangle_{n}\left(t_{1}\right)\right)-B_{n}\left(t_{1}\right)\right\| \\
& \leq\left\|j f\left(\bar{t},\langle u, v\rangle_{n}(\bar{t})\right)-j f\left(t_{1},\langle u, v\rangle\left(t_{1}\right)\right)\right\| \\
& +\left\|j f\left(t_{1},\langle u, v\rangle\left(t_{1}\right)\right)-j f\left(t_{1},\langle u, v\rangle_{n}\left(t_{1}\right)\right)\right\|+2 \varepsilon_{n} \\
& =d_{\infty}^{n}\left(f\left(\bar{t},\langle u, v\rangle_{n}(\bar{t})\right), f\left(t_{1},\langle u, v\rangle\left(t_{1}\right)\right)\right) \\
& +d_{\infty}^{n}\left(f\left(t_{1},\langle u, v\rangle\left(t_{1}\right)\right), f\left(t_{1},\langle u, v\rangle_{n}\left(t_{1}\right)\right)\right)+2 \varepsilon_{n} \\
& <\frac{\varepsilon}{4}+\frac{\varepsilon}{4}+2 \varepsilon_{n}<\varepsilon
\end{aligned}
$$


whenever $n>N$ and $t_{1}<t<t_{1}+\delta$.

Now let $n \longrightarrow \infty$, and applying Eq. (3.5), we have

$$
\left\|\frac{j\langle u, v\rangle(t)-j\langle u, v\rangle\left(t_{1}\right)}{t-t_{1}}-j f\left(t_{1},\langle u, v\rangle\left(t_{1}\right)\right)\right\| \leq \varepsilon, \quad t_{1}<t<t_{1}+\delta .
$$

On the other hand, from the assumption of Theorem 3.1, there exists an $\delta\left(t_{1}\right) \in(0, \delta)$ such that the H-differences

$$
\langle u, v\rangle_{n}(t) \ominus\langle u, v\rangle_{n}\left(t_{1}\right)
$$

exist for all $t \in\left[t_{1}, t_{1}+\delta\left(t_{1}\right)\right]$ and $n=1,2, \ldots$

Let $\langle z, w\rangle_{n}(t)=\langle u, v\rangle_{n}(t) \ominus\langle u, v\rangle_{n}\left(t_{1}\right)$. We verify that the intuitionistic fuzzy number-valued sequence $\left\{\langle z, w\rangle_{n}(t)\right\}$ uniformly converges on $\left[t_{1}, t_{1}+\delta\left(t_{1}\right)\right]$

In fact, from the assumption $d_{\infty}^{n}\left(\langle u, v\rangle_{n}(t),\langle u, v\rangle(t)\right) \longrightarrow 0$, u.c $\forall t \in\left[t_{0}, t_{0}+r\right]$, we know

$$
\begin{aligned}
d_{\infty}^{n}\left(\langle z, w\rangle_{n}(t),\langle z, w\rangle_{m}(t)\right) & =d_{\infty}^{n}\left(\langle z, w\rangle_{n}(t)+\langle u, v\rangle_{n}\left(t_{1}\right),\langle z, w\rangle_{m}(t)+\langle u, v\rangle_{n}\left(t_{1}\right)\right) \\
& \leq d_{\infty}^{n}\left(\langle u, v\rangle_{n}(t),\langle u, v\rangle_{m}(t)\right) \\
& +d_{\infty}^{n}\left(\langle u, v\rangle_{m}(t),\langle z, w\rangle_{m}(t)+\langle u, v\rangle_{n}\left(t_{1}\right)\right) \\
& =d_{\infty}^{n}\left(\langle u, v\rangle_{n}(t),\langle u, v\rangle_{m}(t)\right) \\
& +d_{\infty}^{n}\left(\langle z, w\rangle_{m}(t)+\langle u, v\rangle_{m}\left(t_{1}\right),\langle z, w\rangle_{m}(t)+\langle u, v\rangle_{n}\left(t_{1}\right)\right) \\
& =d_{\infty}^{n}\left(\langle u, v\rangle_{n}(t),\langle u, v\rangle_{m}(t)\right)+d_{\infty}^{n}\left(\langle u, v\rangle_{m}\left(t_{1}\right),\langle u, v\rangle_{n}\left(t_{1}\right)\right) \\
& \longrightarrow 0 u . c \quad \forall t \in\left[t_{1}, t_{1}+\delta\left(t_{1}\right)\right](n, m \longrightarrow \infty)
\end{aligned}
$$

Since $\left(I F_{n}, d_{\infty}^{n}\right)$ is complete, there exists an intuitionistic fuzzy number-valued mapping such that $\left\{\langle z, w\rangle_{n}(t)\right\}$ uniformly converges to $\langle z, w\rangle(t)$ on $\left[t_{1}, t_{1}+\delta\left(t_{1}\right)\right]$ as $n \longrightarrow \infty$

In addition, we have

$$
\begin{aligned}
d_{\infty}^{n}\left(\langle u, v\rangle\left(t_{1}\right)+\langle z, w\rangle(t),\langle u, v\rangle(t)\right) & \leq d_{\infty}^{n}\left(\langle u, v\rangle\left(t_{1}\right)+\langle z, w\rangle(t),\langle u, v\rangle_{n}\left(t_{1}\right)+\langle z, w\rangle_{n}(t)\right) \\
& +d_{\infty}^{n}\left(\langle u, v\rangle_{n}\left(t_{1}\right)+\langle z, w\rangle_{n}(t),\langle u, v\rangle(t)\right) \\
& \leq d_{\infty}^{n}\left(\langle u, v\rangle\left(t_{1}\right)+\langle z, w\rangle(t),\langle u, v\rangle\left(t_{1}\right)+\langle z, w\rangle_{n}(t)\right) \\
& +d_{\infty}^{n}\left(\langle u, v\rangle\left(t_{1}\right)+\langle z, w\rangle_{n}(t),\langle u, v\rangle_{n}\left(t_{1}\right)+\langle z, w\rangle_{n}(t)\right) \\
& +d_{\infty}^{n}\left(\langle u, v\rangle_{n}\left(t_{1}\right),\langle u, v\rangle(t)\right) \\
& =d_{\infty}^{n}\left(\langle z, w\rangle_{n}(t),\langle z, w\rangle(t)\right)+d_{\infty}^{n}\left(\langle u, v\rangle_{n}\left(t_{1}\right),\langle u, v\rangle\left(t_{1}\right)\right) \\
& +d_{\infty}^{n}\left(\langle u, v\rangle_{n}(t),\langle u, v\rangle(t)\right)
\end{aligned}
$$

$\forall t \in\left[t_{1}, t_{1}+\delta\left(t_{1}\right)\right]$

Let $n \longrightarrow \infty$. It follows that

$$
\langle u, v\rangle\left(t_{1}\right) \oplus\langle z, w\rangle(t) \equiv\langle u, v\rangle(t) \quad \text { for all } \quad t \in\left[t_{1}, t_{1}+\delta\left(t_{1}\right)\right]
$$


Hence the H-differences $\langle u, v\rangle(t) \ominus\langle u, v\rangle\left(t_{1}\right)$ exist for all $t_{1} \in\left[t_{1}, t_{1}+\delta\left(t_{1}\right)\right]$

Thus from (3.11) we have

$$
d_{\infty}^{n}\left(\frac{\langle u, v\rangle(t) \ominus\langle u, v\rangle\left(t_{1}\right)}{t-t_{1}}, f\left(t_{1},\langle u, v\rangle\left(t_{1}\right)\right)\right) \leq \varepsilon, \quad t_{1}<t \leq t_{1}+\delta\left(t_{1}\right)
$$

So $\lim _{t \rightarrow t_{1}^{+}} \frac{\langle u, v\rangle(t) \ominus\langle u, v\rangle\left(t_{1}\right)}{t-t_{1}}=f\left(t_{1},\langle u, v\rangle\left(t_{1}\right)\right)$. Similarly, we have

$$
\lim _{t \rightarrow t_{1}^{-}} \frac{\langle u, v\rangle(t) \ominus\langle u, v\rangle\left(t_{1}\right)}{t-t_{1}}=f\left(t_{1},\langle u, v\rangle\left(t_{1}\right)\right)
$$

Hence $\langle u, v\rangle^{\prime}\left(t_{1}\right)$ exists and

$$
\langle u, v\rangle^{\prime}\left(t_{1}\right)=f\left(t_{1},\langle u, v\rangle\left(t_{1}\right)\right)
$$

From $t_{1} \in\left[t_{0}, t_{0}+r\right]$ is arbitrary, we know that Eq. (3.4) holds true and

$$
\langle u, v\rangle \in C^{1}\left[\left[t_{0}, t_{0}+r\right], B\left(\langle u, v\rangle_{0}, q\right)\right] .
$$

Thus, we conclude the proof.

Corollary 3.1. If we replace condition (3.2) by

$$
\begin{array}{r}
j\langle u, v\rangle_{n+1}^{\prime}(t)=j f\left(t,\langle u, v\rangle_{n}(t)\right)+B_{n}(t),\langle u, v\rangle\left(t_{0}\right)=\langle u, v\rangle_{0}, \\
\left\|B_{n}(t)\right\| \leq \varepsilon_{n} \forall t \in\left[t_{0}, t_{0}+r\right](n=0,1, \ldots)
\end{array}
$$

and retain other assumptions, then the conclusions also hold true.

Proof 2. This is completely similar to the proof of Theorem 3.1.

\section{Existence and uniqueness theorem for a solution}

\section{Theorem 4.1. Let}

(a) $f \in C\left[R_{0}, I F_{n}\right]$ and $d_{\infty}^{n}\left(f(t,\langle u, v\rangle), 0_{(1,0)}\right) \leq M$ for all $(t,\langle u, v\rangle) \in R_{0}$.

(b) $g \in C\left[\left[t_{0}, t_{0}+p\right] \times[0, q], R\right], g(t, 0) \equiv 0$ and $g(t, x) \leq M_{1}$, for all $t \in\left[t_{0}, t_{0}+p\right], 0 \leq x \leq q$ such that $g(t, x)$ is nondecreasing on $x$ (i.e, $t_{0} \leq t \leq t_{0}+p, 0 \leq x_{1} \leq x_{2} \leq q \Longrightarrow$ $\left.g\left(t, x_{1}\right) \leq g\left(t, x_{2}\right)\right)$, the initial value problem

$$
x^{\prime}(t)=g(t, x(t)), \quad x\left(t_{0}\right)=0
$$

has only the solution $x(t) \equiv 0$ on $\left[t_{0}, t_{0}+p\right]$.

(c) $d_{\infty}^{n}\left(f(t,\langle u, v\rangle), f\left(t,\left\langle u^{\prime}, v^{\prime}\right\rangle\right)\right) \leq g\left(t, d_{\infty}^{n}\left(\langle u, v\rangle,\left\langle u^{\prime}, v^{\prime}\right\rangle\right)\right)$, for all $(t,\langle u, v\rangle),\left(t,\left\langle u^{\prime}, v^{\prime}\right\rangle\right) \in$ $R_{0}$, and $d_{\infty}^{n}\left(\langle u, v\rangle,\left\langle u^{\prime}, v^{\prime}\right\rangle\right) \leq q$. 
Then the Cauchy problem (3.4) has a unique solution $\langle u, v\rangle \in C^{1}\left[\left[t_{0}, t_{0}+r\right], B\left(x_{0}, q\right)\right]$ on $\left[t_{0}, t_{0}+r\right]$, where $r=\min \left\{p, q / M, q / M_{1}\right\}$, and the successive iterations

$$
\langle u, v\rangle_{0}(t)=\langle u, v\rangle_{0},\langle u, v\rangle_{n+1}(t)=\langle u, v\rangle_{0} \oplus \int_{t_{0}}^{t} f\left(s,\langle u, v\rangle_{n}(s)\right) d s \quad(n=0,1,2, \ldots)
$$

uniformly converge to $\langle u, v\rangle(t)$ on $\left[t_{0}, t_{0}+r\right]$.

Proof 3. from (4.2) and the assumption (a), by the inductive method we know

$$
\begin{aligned}
d_{\infty}^{n}\left(\langle u, v\rangle_{n+1}(t),\langle u, v\rangle_{0}\right) & \leq \int_{t_{0}}^{t} d_{\infty}^{n}\left(f\left(s,\langle u, v\rangle_{n}(s)\right), 0_{(1,0)}\right) d s \\
& \leq q \forall t \in\left[t_{0}, t_{0}+r\right] \quad n=0,1,2, \ldots
\end{aligned}
$$

Hence $\langle u, v\rangle_{n+1} \in C^{1}\left[\left[t_{0}, t_{0}+r\right], B\left(x_{0}, q\right)\right]$ and

$$
\langle u, v\rangle_{n+1}^{\prime}(t)=f\left(t,\left(\langle u, v\rangle_{n}(t)\right), \quad\langle u, v\rangle_{n}\left(t_{0}\right)=\langle u, v\rangle_{0} \quad(n=0,1,2, \ldots)\right.
$$

Let $M_{2}=\max \left\{M, M_{1}\right\}$. Then $r=\min \left\{p, q / M_{2}\right\}$. and we get the successive iterations as

$$
\left\{\begin{array}{l}
x_{0}(t)=\quad M_{2}\left(t-t_{0}\right) \quad t_{0} \leq t \leq t_{0}+r \\
x_{n+1}(t)=\int_{t_{0}}^{t} g\left(s, x_{n}(s)\right), \quad t_{0} \leq t \leq t_{0}+r \quad(n=0,1,2, \ldots)
\end{array}\right.
$$

It is immediate that

$$
x_{1}(t)=\int_{t_{0}}^{t} g\left(s, x_{0}(s)\right) \leq M_{1}\left(t-t_{0}\right) \leq x_{0}(t) \leq q, \quad \forall t \in\left[t_{0}, t_{0}+r\right]
$$

So, by the inductive method and in view that $g(t, x)$ is nondecreasing on $x$, we have

$$
0 \leq x_{n+1}(t) \leq x_{n}(t) \leq q, \quad \forall t \in\left[t_{0}, t_{0}+r\right] \quad(n=0,1,2, \ldots)
$$

As $\left|x_{n+1}^{\prime}(t)\right|=\left|g\left(t, x_{n}(t)\right)\right| \leq M_{1}$, from the Ascoli-Arzela theorem and (4.7) we know that $\left\{x_{n}(t)\right\}$ uniformly converges to some continuous function $x(t)$ on $\left[t_{0}, t_{0}+r\right]$ and

$$
x(t)=\int_{t_{0}}^{t} g(s, x(s)) d s .
$$

Thus $x \in C^{1}\left[\left[t_{0}, t_{0}+r\right],[0, q]\right]$ and $x$ is the solution the initial value problem (4.1). From assumption (b) we get $x(t) \equiv 0$. In addition, we have

$$
\begin{aligned}
& d_{\infty}^{n}\left(\langle u, v\rangle_{1}(t),\langle u, v\rangle_{0}\right)=d_{\infty}^{n}\left(\int_{t_{0}}^{t} f\left(s,\langle u, v\rangle_{0}(s)\right) d s, 0_{(1,0)}\right) \\
& \leq \int_{t_{0}}^{t} d_{\infty}^{n}\left(f\left(s,\langle u, v\rangle_{0}(s)\right), 0_{(1,0)}\right) d s \leq M\left(t-t_{0}\right) \leq x_{0}(t)
\end{aligned}
$$

Suppose $d_{\infty}^{n}\left(\langle u, v\rangle_{k}(t),\langle u, v\rangle_{k-1}\right) \leq x_{k-1}(t)$, then by the assumption (c), we have

$$
\begin{aligned}
& d_{\infty}^{n}\left(\langle u, v\rangle_{k+1}(t),\langle u, v\rangle_{k}(t)\right)=d_{\infty}^{n}\left(\int_{t_{0}}^{t} f\left(s,\langle u, v\rangle_{k}(s)\right) d s, \int_{t_{0}}^{t} f\left(s,\langle u, v\rangle_{k-1}(s)\right) d s\right) \\
& \leq \int_{t_{0}}^{t} d_{\infty}^{n}\left(f\left(s,\langle u, v\rangle_{k}(s)\right), f\left(s,\langle u, v\rangle_{k-1}(s)\right)\right) d s \leq \int_{t_{0}}^{t} g\left(s, d_{\infty}^{n}\left(\langle u, v\rangle_{k}(s),\langle u, v\rangle_{k-1}(s)\right)\right) d s \\
& \leq \int_{t_{0}}^{t} g\left(s, x_{k-1}(s)\right) d s=x_{k}(t)
\end{aligned}
$$


Thus by the inductive method we know

$$
d_{\infty}^{n}\left(\langle u, v\rangle_{n+1}(t),\langle u, v\rangle_{n}(t)\right) \leq x_{n}(t) \quad t_{0} \leq t \leq t_{0}+r \quad(n=0,1,2, \ldots) .
$$

So, we have

$$
\begin{aligned}
d_{\infty}^{n}\left(\langle u, v\rangle_{n+1}^{\prime}(t),\langle u, v\rangle_{n}^{\prime}(t)\right) & =d_{\infty}^{n}\left(f\left(t,\langle u, v\rangle_{n}(t)\right), f\left(t,\langle u, v\rangle_{n-1}(t)\right)\right) \\
& \leq g\left(t, d_{\infty}^{n}\left(\langle u, v\rangle_{n}(t),\langle u, v\rangle_{n-1}(t)\right)\right) \\
& \leq g\left(t, x_{n-1}(t)\right)
\end{aligned}
$$

Assume $m \geq n$, and in view of (4.9) and (4.7) we get

$$
\begin{aligned}
d_{\infty}^{n}\left(\langle u, v\rangle_{n}^{\prime}(t),\langle u, v\rangle_{m}^{\prime}(t)\right) & \leq d_{\infty}^{n}\left(f\left(t,\langle u, v\rangle_{n-1}(t)\right), f\left(t,\langle u, v\rangle_{n}(t)\right)\right) \\
& +d_{\infty}^{n}\left(f\left(t,\langle u, v\rangle_{n}(t)\right), f\left(t,\langle u, v\rangle_{m}(t)\right)\right) \\
& +d_{\infty}^{n}\left(f\left(t,\langle u, v\rangle_{m}(t)\right), f\left(t,\langle u, v\rangle_{m-1}(t)\right)\right) \\
& \leq g\left(t, x_{n-1}(t)\right)+g\left(t, d_{\infty}^{n}\left(\langle u, v\rangle_{n}(t),\langle u, v\rangle_{m}(t)\right)\right)+g\left(t, x_{m-1}(t)\right) \\
& \leq 2 g\left(t, x_{n-1}(t)\right)+g\left(t, d_{\infty}^{n}\left(\langle u, v\rangle_{n}(t),\langle u, v\rangle_{m}(t)\right)\right)
\end{aligned}
$$

Furthermore, from

$$
\begin{aligned}
& \left.d_{\infty}^{n}\left(\langle u, v\rangle_{n}(t+h),\langle u, v\rangle_{m}(t+h)\right)\right) \\
& \left.\leq d_{\infty}^{n}\left(\langle u, v\rangle_{n}(t+h),\langle u, v\rangle_{m}(t+h)-\langle u, v\rangle_{m}(t)+\langle u, v\rangle_{n}(t)\right)\right) \\
& \left.\left.+d_{\infty}^{n}\left(\langle u, v\rangle_{m}(t+h) \ominus\langle u, v\rangle_{m}(t)+\langle u, v\rangle_{n}(t)\right),\langle u, v\rangle_{m}(t+h)\right)\right) \\
& \left.=d_{\infty}^{n}\left(\langle u, v\rangle_{n}(t+h) \ominus\langle u, v\rangle_{n}(t),\langle u, v\rangle_{m}(t+h) \ominus\langle u, v\rangle_{m}(t)\right)\right) \\
& \left.+d_{\infty}^{n}\left(\langle u, v\rangle_{n}(t),\langle u, v\rangle_{m}(t)\right)\right)
\end{aligned}
$$

we deduce that

$$
\begin{aligned}
& \left.D^{+} d_{\infty}^{n}\left(\langle u, v\rangle_{n}(t),\langle u, v\rangle_{m}(t)\right)\right) \\
& =\varlimsup_{h \rightarrow 0^{+}} \frac{\left.d_{\infty}^{n}\left(\langle u, v\rangle_{n}(t+h),\langle u, v\rangle_{m}(t+h)\right)\right)-d_{\infty}^{n}\left(\langle u, v\rangle_{n}(t),\langle u, v\rangle_{m}(t)\right)}{h} \\
& \leq \varlimsup_{h \rightarrow 0^{+}} \frac{\left.d_{\infty}^{n}\left(\langle u, v\rangle_{n}(t+h) \ominus\langle u, v\rangle_{n}(t),\langle u, v\rangle_{m}(t+h)\right) \ominus\langle u, v\rangle_{m}(t)\right)}{h} \\
& \left.=d_{\infty}^{n}\left(\langle u, v\rangle_{n}^{\prime}(t),\langle u, v\rangle_{m}^{\prime}(t)\right)\right)<2 g\left(t, x_{n-1}(t)\right)+g\left(t, d_{\infty}^{n}\left(\langle u, v\rangle_{n}(t),\langle u, v\rangle_{m}(t)\right)\right)
\end{aligned}
$$

Since $g\left(t, x_{n-1}(t)\right)$ uniformly converges to 0 , then for arbitrary $\varepsilon>0$ there exists a natural number $N$ such that

$$
\left.D^{+} d_{\infty}^{n}\left(\langle u, v\rangle_{n}(t),\langle u, v\rangle_{m}(t)\right)\right)<g\left(t, d_{\infty}^{n}\left(\langle u, v\rangle_{n}(t),\langle u, v\rangle_{m}(t)\right)\right)+\varepsilon \quad \forall m \geq n>N
$$


Here $D^{+}$is the Dini derivative (see [7]). From the fact that $d_{\infty}^{n}\left(\langle u, v\rangle_{n}\left(t_{0}\right),\langle u, v\rangle_{m}\left(t_{0}\right)\right)=0<\varepsilon$ and by proposition 2.1 , we have

$$
\left.d_{\infty}^{n}\left(\langle u, v\rangle_{n}(t),\langle u, v\rangle_{m}(t)\right)\right) \leq w(t, \varepsilon) \quad \forall \in\left[t_{0}, t_{0}+r\right] \quad \forall m \geq n>N
$$

where $w(t, \varepsilon)$ is the maximum solution to the initial value problem

$$
x^{\prime}(t)=g(t, x(t))+\varepsilon, \quad x\left(t_{0}\right)=\varepsilon
$$

By proposition 2.2 we know that $w(t, \varepsilon)$ uniformly converges to the maximum solution $x(t) \equiv 0$ of problem (4.1) on $t_{0} \leq t \leq t_{0}+r$ as $\varepsilon \rightarrow 0$.

Thus, according to (4.10) and that $\left(I F_{n}, d_{\infty}^{n}\right)$ is complete, we know that there exists an intuitionistic fuzzy set-valued mapping $\langle u, v\rangle: T \longrightarrow I F_{n}$ such that $\left.d_{\infty}^{n}\left(\langle u, v\rangle_{n}(t),\langle u, v\rangle(t)\right)\right)$ uniformly converges to 0 as $n \longrightarrow \infty$. Applying (4.4) and Corollary (3.1) we have $\langle u, v\rangle \in$ $C^{1}\left[\left[t_{0}, t_{0}+r\right], B\left(\langle u, v\rangle_{0}, q\right)\right]$ and $\langle u, v\rangle(t)$ is the solution of the initial value problem (3.4).

Finally, we prove the uniqueness. Suppose $\langle z, w\rangle(t)$ is another solution of initial value problem (3.4). Let

$$
m(t)=d_{\infty}^{n}(\langle u, v\rangle(t),\langle z, w\rangle(t))
$$

Then $m\left(t_{0}\right) \equiv 0$

$$
D^{+} m(t) \leq d_{\infty}^{n}\left(\langle u, v\rangle^{\prime}(t),\langle z, w\rangle^{\prime}(t)\right)=d_{\infty}^{n}(f(t,\langle u, v\rangle(t)), f(t,\langle z, w\rangle(t))) \leq g(t, m(t)) .
$$

Hence from proposition 2.2 we know

$$
d_{\infty}^{n}(\langle u, v\rangle(t),\langle z, w\rangle(t)) \leq x(t) \equiv 0, \quad \forall \in\left[t_{0}, t_{0}+r\right]
$$

where $x(t) \equiv 0$ is the maximum solution of problem (4.1) on $\left[t_{0}, t_{0}+r\right]$.

Therefore $\langle u, v\rangle(t)=\langle z, w\rangle(t)$.

Corollary 4.1. Let $f \in C\left[R_{0}, I F_{n}\right]$ such that $d_{\infty}^{n}(f(t,\langle u, v\rangle), 0) \leq M$ for all $(t,\langle u, v\rangle) \in R_{0}$ and $f$ satisfies the Lipschitz condition

$$
d_{\infty}^{n}\left(f(t,\langle u, v\rangle), f\left(t,\left\langle u^{\prime}, v^{\prime}\right\rangle\right)\right) \leq L d_{\infty}^{n}\left(\langle u, v\rangle,\left\langle u^{\prime}, v^{\prime}\right\rangle\right), \quad \forall(t,\langle u, v\rangle),(t,\langle u, v\rangle) \in R_{0}
$$

where $L$ is a constant. Then the Cauchy problem (3.4) has an unique solution $\langle u, v\rangle \in C^{1}\left[\left[t_{0}, t_{0}+\right.\right.$ $\left.r], B\left(x_{0}, q\right)\right]$ on $\left[t_{0}, t_{0}+r\right]$, where $r=\min \{p, q / M, 1 / L\}$, and the successive iterations (4.2) uniformly converge to $\langle u, v\rangle(t)$ on $\left[t_{0}, t_{0}+r\right]$.

Proof 4. In the proof of Theorem (4.1), taking $g(t, x)=L . x$ we then obtain the proof of Corollary 4.1 , where $M_{1}=L . q$, hence $r=\min \{p, q / M, 1 / L\}$.

\section{References}

[1] Atanassov, K. (1983) Intuitionistic Fuzzy Sets, VII ITKR Session, Sofia, 20-23 June 1983 (Deposed in Centr. Sci.-Techn. Library of the Bulg. Acad. of Sci., 1697/84) (in Bulgarian). Reprinted: Int. J. Bioautomation, 2016, 20(S1), S1-S6. 
[2] Atanassov, K. (1986) Intuitionistic fuzzy sets, Fuzzy Sets and Systems, 20(1), 87-96.

[3] Atanassov, K. (1994) Operators over interval valued intuitionistic fuzzy sets, Fuzzy Sets and Systems, 64(2), 159-174.

[4] De, S. K., Biswas, R., \& Roy, A. R. (2001) An application of intuitionistic fuzzy sets in medical diagnosis, Fuzzy Sets and Systems, 117(2), 209-213.

[5] El Allaoui, A., Melliani, S., \& Chadli, L.S. (2016) The Cauchy problem for complex intuitionistic fuzzy differential equations, Notes on Intuitionistic Fuzzy Sets, 22(4), 53-63.

[6] Kharal, A. (2009) Homeopathic drug selection using intuitionistic fuzzy sets, Homeopathy, 98(1), 35-39.

[7] Lakshmikantham, V., \& Leela, S. (1969) Differential and Integral Inequalities, Vols. I and II, Academic Press, New York.

[8] Li, D.F. \& Cheng, C. T. (2002) New similarity measures of intuitionistic fuzzy sets and application to pattern recognitions, Pattern Recognit. Lett., 23(1-3), 221-225.

[9] Li, D. F. (2005) Multiattribute decision making models and methods using intuitionistic fuzzy sets, J. Comput. Syst. Sci., 70, 73-85.

[10] Klement, E. P., Puri, M. L., \& Ralescu, D. A. (1986) Limit Theorems for Fuzzy Random Variables, Proc. R. Soc. Lond. A, 407, 171-182.

[11] Melliani, S., \& Chadli, L. S. (2000) Intuitionistic fuzzy differential equation. Part 1, Notes on Intuitionistic Fuzzy Sets, 6(2), 37-41.

[12] Melliani, S., Elomari, M., Chadli, L. S., \& Ettoussi, R. (2015) Intuitionistic Fuzzy Metric Space, Notes on Intuitionistic Fuzzy sets, 21(1), 43-53.

[13] Melliani, S., Elomari, M., Chadli, L. S., \& Ettoussi, R. (2015) Intuitionistic fuzzy fractional equation, Notes on Intuitionistic Fuzzy Sets, 21(4), 76-89.

[14] Wu, C., \& Song, S. (1996) Approximate Solutions, Existence, and Uniqueness of the Cauchy Problem of Fuzzy Differential Equations, Journal Of Mathematical Analysis And Applications, 202, 629-644.

[15] Zadeh, L. A. (1965) Fuzzy sets, Inf. Control, 8, 338-353. 